\title{
L'évolution des bases sociales du mouvement des caisses Desjardins. Le sociétariat de la Fédération régionale du centre du Québec (1909-1965)
}

\section{Roger Levasseur et Yvan Rousseau}

Volume 45, numéro 3, hiver 1992

URI : https://id.erudit.org/iderudit/304990ar

DOI : https://doi.org/10.7202/304990ar

Aller au sommaire du numéro

Éditeur(s)

Institut d'histoire de l'Amérique française

ISSN

0035-2357 (imprimé)

1492-1383 (numérique)

Découvrir la revue

Citer cet article

Levasseur, R. \& Rousseau, Y. (1992). L'évolution des bases sociales du mouvement des caisses Desjardins. Le sociétariat de la Fédération régionale du centre du Québec (1909-1965). Revue d'histoire de l'Amérique française, 45(3), 343-374. https://doi.org/10.7202/304990ar

\section{Résumé de l'article}

Partant d'une analyse des bases sociales et territoriales des Caisses Desjardins dans la région du Centre du Québec, les auteurs tentent de dégager les lignes de forces de leur évolution jusqu'au milieu des années 1960. L'étude met au jour les relations privilégiées qu'elles ont entretenues avec la paroisse, le monde agricole et l'ancienne petite bourgeoisie pendant le premier tiers du siècle. La Seconde Guerre a été l'occasion pour le mouvement des caisses d'entreprendre le virage imposé par la croissance économique et démographique du front urbain, d'élargir ses bases sociales et d'étendre son réseau d'établissements à l'échelle de la région. Appuyée désormais sur la croissance des villes, l'évolution du mouvement sera marquée par un approfondissement des écarts de développement entre les caisses, l'accession de nouveaux groupes à sa direction et un effritement de ses rapports avec la paroisse, sa communauté d'origine.
Tous droits réservés Institut d'histoire de l'Amérique française, 1992
Ce document est protégé par la loi sur le droit d'auteur. L'utilisation des services d'Érudit (y compris la reproduction) est assujettie à sa politique d'utilisation que vous pouvez consulter en ligne. 


\title{
L'ÉVOLUTION DES BASES SOCIALES DU MOUVEMENT DES CAISSES DESJARDINS LE SOCIÉTARIAT DE LA FÉDÉRATION RÉGIONALE DU CENTRE DU QUÉBEC (1909-1965) ${ }^{1}$
}

\section{RÉSUMÉ}

\author{
ROGER LEVASSEUR \\ YVAN ROUSSEAU \\ Centre d'études québécoises \\ Université du Québec à Trois-Rivières
}

Partant d'une analyse des bases sociales et territoriales des Caisses Desjardins dans la région du Centre du Québec, les auteurs tentent de dégager les lignes de forces de leur évolution jusqu'au milieu des années 1960. L'étude met au jour les relations privilégiées qu'elles ont entretenues avec la paroisse, le monde agricole et l'ancienne petite bourgeoisie pendant le premier tiers du siècle. La Seconde Guerre a été l'occasion pour le mouvement des caisses d'entreprendre le virage imposé par la croissance économique et démographique du front urbain, d'élargir ses bases sociales et d'étendre son réseau d'établissements à l'échelle de la région. Appuyée désormais sur la croissance des villes, l'évolution du mouvement sera marquée par un approfondissement des écarts de développement entre les caisses, l'accession de nouveaux groupes à sa direction et un effritement de ses rapports avec la paroisse, sa communauté d'origine.

\begin{abstract}
Starting from an analysis of the social and territorial bases of the Caisses Desjardins within the heartland of Quebec, the authors have undertaken to trace the main lines of the Caisses' development up to the mid 1960s. This study brings up to date the special relationship which the Caisses maintained with local parishes, with the agricultural sector and with the «petite bourgeoisie» during the first third of the century. The Second World War, however, facilitated a movement allowing the Caisses to follow a natural economic and demographic expansion on the urban front, to broaden their social base and to extend their network throughout the region. Supported thenceforth by urban expansion, the movement was to be characterized by a deepening of the discrepancy in the relative development of different Caisses, by a jockeying for position among the new groups at the helm and by a gradual erosion of the Caisse's links with the parishes, which first fostered the movement.
\end{abstract}

1 La présente étude, qui s'inscrit dans le cadre d'un programme de recherche sur l'histoire sociale du mouvement Desjardins dans la région de la Mauricie et des Bois-Francs, a reçu un soutien financier du CRSHC, du Mouvement Desjardins (La Fédération des caisses populaires Desjardins du Centre du Québec et la Confédération des caisses populaires et d'économie Desjardins du Québec) et de l'Université du Québec à Trois-Rivières. Quelques étudiants ont participé à la collecte des données. Ce sont Clémence Bélanger, Mireille Lehoux, Alain Ruest et Gilles Vallée. Nous tenons à remercier nos collègues Serge Gagnon, Normand Séguin et Paul Sabourin ainsi que Pierre Poulin, de la Société historique Alphonse-Desjardins, pour leurs commentaires.

RHAF, vol. 45, n 3, hiver 1992 
Durant la seconde moitié du XIX $\mathrm{X}^{\mathrm{e}}$ siècle, les coopératives de crédit et les banques populaires se multiplient sur le continent européen. Si les formes d'organisation et les services dispensés par ces mutuelles sont multiples, toutes se rejoignent sur la nécessité d'élargir l'accès au crédit par le recours à l'association coopérative. Elles s'inspirent de deux grandes traditions: le christianisme social et le socialisme. Les coopératives québécoises s'inscrivent davantage dans la tradition chrétienne. La convergence entre mutualité et catholicisme culminera avec l'appel de Léon XIII dans son encyclique Rerum Novarum en 1891 invitant les ecclésiastiques et les laïcs à propager les œuvres sociales. Il préconise la formation de sociétés de secours mutuels, d'organisations professionnelles et d'associations pour désamorcer les conflits sociaux et favoriser une réforme sociale basée sur l'application des valeurs chrétiennes.

Au Québec, la mutualité d'épargne et de crédit est introduite au tout début du $\mathrm{XX}^{\mathrm{e}}$ siècle par Alphonse Desjardins, ancien journaliste devenu sténographe à la Chambre des Communes. Après quelques années consacrées à l'étude de la coopération et à la mise au point d'un modèle adapté à la réalité du Québec, il fonde la première caisse populaire à Lévis en 1900. De son vivant, 186 autres caisses d'épargne et de crédit, relativement dispersées et jouissant d'une autonomie à peu près complète, seront organisées dans la province sans que soit réalisé son projet de fédération et d'établissement d'une caisse centrale $^{2}$. Le 15 décembre 1920 , soit deux mois après le décès du pionnier lévisien, une douzaine de caisses du diocèse de TroisRivières procèdent à la fondation de la première Union régionale de caisses populaires, contrecarrant ainsi les initiatives en provenance de Lévis en faveur de la formation d'une fédération provinciale de caisses.

Dans le cadre de cet article, nous examinons les grandes transformations du sociétariat ${ }^{3}$. L'Union régionale de Trois-Rivières et ses caisses affiliées circonscrivent le territoire de notre enquête depuis la fondation des premiers établissements en 1909 jusqu'à la Révolution

2 Pierre Poulin, Histoire du Mouvement Desjardins, tome 1: Desjardins et la naissance des caisses populaires 1900-1920 ([Montréal], Québec/Amérique, collection Desjardins, 1990), 152.

3 Cet article se situe dans une démarche plus large. Voir Roger Levasseur, «Vers une histoire sociale du mouvement des caisses Desjardins en Mauricie: de ses débuts à nos jours», Coopératives et développement, 22,2 (1991). Certains résultats touchant les politiques de crédit, la gérance locale et le développement inégal du mouvement ont été abordés sommairement; voir Michel Bellefleur, Roger Levasseur et Yvan Rousseau, «La libéralisation du crédit dans le mouvement des caisses Desjardins», Les stratégies culturelles au Québec $\left(19^{\circ}-20^{*}\right)$, à paraitre à l'Institut québécois de recherche sur la culture dans les Actes du colloque organisé par le Centre d'études québécoises, Trois-Rivières, novembre 1990. 
tranquille ${ }^{4}$. Établies sur les territoires bornés par les limites des diocèses de Trois-Rivières (depuis 1920), sur la rive nord du SaintLaurent, et de Nicolet (depuis 1922), sur sa rive opposée, elles offrent un assortiment de situations suffisamment varié pour que les résultats de nos travaux soient révélateurs de l'évolution que connaît le mouvement des caisses dans son ensemble. En effet, la structuration de la région de la Mauricie et des Bois-Francs au $\mathrm{XX}^{\mathrm{e}}$ siècle va s'appuyer sur quatre pôles de développement urbain et industriel de relative importance, quelques petites villes, axées tantôt sur l'agriculture et tantôt sur l'industrie, et un bon nombre de localités rurales à vocation plutôt agricole 5 .

Le développement des caisses populaires obéit à deux rationalités étroitement liées qui font sa singularité: celle de l'association que forment ses membres et celle de l'entreprise financière dont la croissance est soumise aux règles de l'économie de marché. En tant que coopérative, la caisse est, selon la formule de Claude Vienney, «la combinaison d'un regroupement de personnes et d'une entreprise réciproquement liés par un rapport d'activité et de sociétariat» ${ }^{6}$. Ce qui distingue la caisse de la plupart des coopératives, c'est le fait de recruter ses membres non pas suivant le métier ou la profession, mais à l'intérieur d'un territoire donné, la paroisse. Son projet est de mobiliser la petite épargne, d'organiser un crédit décentralisé et de favoriser ainsi le développement de la localité. Non seulement la caisse

4 Nous avons procédé au dépouillement des Déclarations de fondation et des Procèsverbaux des assemblées de fondation de chacune des caisses locales. L'exploitation systématique des déclarations de fondation nous a permis de recenser, pour chacune des caisses locales, le nom, la profession, l'état civil et le sexe de chacun des fondateurs de caisses de même que le nombre de parts sociales qu'ils souscrivent. Les procès-verbaux des assemblées de fondation et de la première réunion des conseils d'administration sont généralement annexés aux déclarations. Ils ont été mis à profit pour identifier les élus aux trois conseils des caisses. De même, ils ont été utilisés pour cerner les circonscriptions territoriales d'activité de chacune des caisses au moment de leurs fondations. Au total, 143 fondations de caisses sur un total possible de 178, et les informations relatives à 5696 participants incluant 1570 élus locaux ont été recensées. Par ailleurs, les Rapports mensuels de chacune des caisses locales au 31 décembre de l'année ont été exploités sur une base quinquennale depuis 1935 jusqu'en 1965. Avant cette date (1915-1930), nous nous sommes repliés sur les données publiées dans les Annuaires du Québec qui ont fait l'objet d'un traitement similaire.

5 Les agglomérations urbaines de Trois-Rivières-Cap-de-la-Madeleine et ShawiniganGrand-Mère (rive nord du Saint-Laurent), Drummondville, Victoriaville et leur proche périphérie (rive opposée) forment les principales bases du développement urbain de la région étudiée. En marge de ces agglomérations, on trouve quelques petites villes telles que Louiseville, La Tuque et Nicolet. Le reste du territoire régional est formé de campagnes et de villages.

6 Claude Vienney a proposé une problématique intéressante de la formation et des transformations des coopératives. Nous l'utilisons partiellement. Claude Vienney, Socio-économie des organisations coopératives, tome 1: Formation et transformations des institutions $d u$ secteur coopératif français (Paris, CIEM, 1980). 
épouse l'organisation de base de l'Église, mais encore elle compte sur son appui et plus largement sur celui des élites locales pour s'implanter et se développer. Pendant le premier tiers du siècle, les caisses ont entretenu des relations privilégiées avec les agents de la petite production locale; ces derniers ont exercé une influence considérable sur les orientations des caisses en matière d'épargne, de crédit et de placement. Leur enracinement et la percée qu'elles réalisent dans les villes vont modifier sensiblement leurs bases sociales ainsi que leurs rapports à la paroisse. Un processus de «ré-identification des acteurs» s'opère dans l'après-guerre, introduisant un changement majeur sur le plan du sociétariat: on passe du monde de la petite production à celui du salariat. Les sociétaires s'identifieront de plus en plus à la figure du client ou du consommateur, ce qui entraînera une redéfinition des activités de la caisse. Cette nouvelle dynamique traduit l'adaptation du mouvement Desjardins aux nouvelles tendances des marchés. Elle favorise aussi l'affirmation des agents de l'appareil (gérants, spécialistes, employés) au détriment des sociétaires et des élus. Enfin, cette logique de croissance n'est pas étrangère, semble-t-il, à une certaine forme de «délocalisation» des pouvoirs et des activités.

Rendre raison de ces grandes transformations, les examiner à la fois sous l'angle de l'association et de l'entreprise, tel est l'objet de ce texte. Trois temps forts, que nous nous attardons maintenant à reconstituer, ont rythmé l'évolution du mouvement des Caisses Desjardins, de ses origines jusqu'au milieu des années 1960.

\section{1 - LES VICISSITUDES DU DÉMARRAGE (1909-1935)}

Ramenée à sa plus simple expression, la caisse populaire a pour but de canaliser la petite épargne à l'échelle de la localité selon la formule de l'association coopérative, d'en confier la gestion à ses représentants élus et de favoriser l'organisation d'un crédit décentralisé. À travers la mise en place de ces établissements autonomes, Alphonse Desjardins espère notamment revitaliser la petite production agricole, artisanale et industrielle marginalisée par la progression du capitalisme de grande entreprise et la concentration du pouvoir économique.

Le pionnier lévisien, pour l'essentiel, fait reposer l'organisation des caisses sur la paroisse et ses élites. En raison des contacts intimes et nombreux qu'elle favorise entre ses résidants, la paroisse, croit-il, réunit les conditions idéales pour répondre à la double exigence de protéger l'épargne des sociétaires et de s'assurer de la solvabilité et de la moralité des usagers du crédit: 
Par ses relations répétées et constantes de la vie religieuse et municipale comme celle des activités d'ordre matériel ou du voisinage, précise-t-il en 1912, la paroisse offre un champ d'action admirable pour un organe économique dont les opérations sont surtout basées sur la confiance mutuelle, reposant elle-même sur un ensemble de connaissances certaines de ce que vaut chacun des membres de l'association. ${ }^{7}$

Partant d'une vision consensuelle des communautés paroissiales, Desjardins insiste sur la fonction d'harmonisation sociale et économique que la caisse est appelée à jouer dans son milieu ambiant. La caisse populaire, soutient-il en guise de critique à la loi des syndicats agricoles de 1902 qui restreignait leur membership aux seuls agriculteurs, doit favoriser l'«union intime et féconde de toutes les classes [...] vouloir les éloigner, les isoler les unes des autres c'est courir à coup sûr l'inévitable danger de stériliser une partie des énergies...» ${ }^{8}$. Dans son entreprise, Desjardins réserve d'ailleurs un rôle de premier plan aux élites locales qu'il désigne sous les expressions de «principaux citoyens», d' «autorités sociales» ou encore de «classes dirigeantes». Avant tout, Desjardins mise sur la participation du curé, le considérant comme la «principale autorité sociale» de la paroisse. Il compte également sur le concours des administrateurs municipaux et scolaires ainsi que sur «les notaires, les instituteurs, hommes instruits, tout préparés d'avance à aborder avec compétence les devoirs si essentiels à la bonne marche de ces associations» ${ }^{9}$. À maintes reprises, il sollicite leur concours pour introduire et multiplier les caisses, mais aussi pour les diriger: «ces hommes éclairés, amoureux du bien public, pourront non seulement provoquer des créations, mais ils pourront aussi y entrer, les faire fonctionner en acceptant les charges que la confiance de leurs co-sociétaires leur confiera.» ${ }^{10}$

\section{UN MOUVEMENT PRÉCAIRE ET FAIBLEMENT INTÉGRÉ}

À cinq reprises durant l'année 1909, Desjardins vient en Mauricie pour y fonder les premières caisses. Au moment de son décès en octobre 1920, 39 caisses populaires avaient été organisées sur le terri-

7 Alphonse Desjardins, La Caisse populaire (Montréal, L'École sociale populaire, 1912). Les soulignés sont des auteurs.

8 Alphonse Desjardins, «Loi concernant les syndicats coopératifs; exposé des motifs», Cyrille Vaillancourt et Albert Faucher, Alphonse Desjardins. Pionnier de la coopération d'épargne et de crédit en Amérique (Lévis, Le Quotidien, 1950), 106.

9 Alphonse Desjardins, «Mémoire sur l'organisation de l'agriculture dans la province de Québec», Ibidem, 215.

10 Alphonse Desjardins, «Loi concernant les syndicats coopératifs; exposé des motifs», Ibidem, 107. 
toire des diocèses de Trois-Rivières et de Nicolet. Vingt-neuf d'entre elles étaient encore en activité, les autres ayant dû interrompre leurs opérations pour diverses raisons ${ }^{11}$. Un premier examen de ces caisses fait ressortir les traits dominants de leur configuration régionale: elles forment un ensemble à coloration nettement rurale, concentré dans les paroisses des comtés de Champlain et de Saint-Maurice établis sur la rive nord du Saint-Laurent. Les onze caisses actives de la rive sud en 1920 sont disséminées à l'intérieur des quatre comtés formant le diocèse de Nicolet. Quoique Desjardins n'ait jamais explicitement réduit la portée de son entreprise aux milieux ruraux, ce fut son terrain de prédilection. En effet, toutes les caisses actives dans la région de la Mauricie et des Bois-francs en 1920, à l'exception de trois, sont établies dans les campagnes: elles rassemblent $80 \%$ de l'ensemble des sociétaires et disposent des deux tiers de l'actif total du mouvement à l'échelle régionale. Avec un actif global de $840000 \$$ et plus de 4000 sociétaires, les caisses établies sur la rive nord du Saint-Laurent gèrent des fonds cinq fois plus importants et regroupent trois fois plus de membres que leurs consœurs de la rive opposée ${ }^{12}$.

La fédération de caisses qui naît précipitamment aux lendemains du décès d'Alphonse Desjardins n'est pas celle qu'il avait voulu établir à la grandeur du Québec, mais une fédération régionale assise sur les limites territoriales du diocèse. Le 15 décembre 1920, les représentants d'une douzaine de caisses du diocèse de Trois-Rivières procédaient à la fondation de l'Union régionale des caisses populaires de Trois-Rivières (URTR). Les régions de Québec, Montréal et Gaspé allaient emboîter le pas peu de temps après. La nouvelle Union régionale se voyait attribuer en outre les mandats suivants: propager l'idéal de la coopération, fonder de nouvelles caisses, procéder à leur inspection et établir une caisse régionale ${ }^{13}$. D'abord circonscrite au territoire couvert par les comtés fédéraux de Saint-Maurice, Maskinongé et Champlain, l'Union élargissait en 1922 son aire d'activité aux comtés de Nicolet, Yamaska, Arthabaska et Drummond, situés sur la rive sud du Saint-Laurent ${ }^{14}$. La création de l'Union régionale fut

11 La situation des dix caisses inactives en décembre 1920 se présente comme suit: six ont été fondées par A. Desjardins mais n'ont jamais véritablement fonctionné; la caisse de SaintMaurice est paralysée à la suite d'une défalcation en 1920; Yamachiche et Sainte-Gertrude sont fermées en 1918; Saint-Roch-de-Mékinac avait été fermée en 1913, soit un an après sa fondation.

12 Les chiffres ont été obtenus à partir des données publiées dans l'édition de 1921 de l'Annuaire du Québec. 1920.

13 Statuts de l'Union régionale des caisses populaires de Trois-Rivières, 15 décembre

14 Procès-verbal de la séance du Conseil d'administration de l'Union régionale de Trois-Rivières, 27 avril 1922. 
l'expression de la volonté d'un groupe de dirigeants autonomistes de la région de Trois-Rivières. Réfractaires à l'idée d'abandonner la gestion de leurs fonds à une éventuelle caisse provinciale, ils voyaient dans la régionalisation un moindre mal à la centralisation.

Au cours des années 1920, une des principales préoccupations des dirigeants de l'URTR fut d'élargir et de consolider son sociétariat, voire d'《occuper» son territoire de juridiction. À cet égard, elle se buta à l'opposition de plusieurs caisses qui, jouant la carte de l'autonomie, étaient hostiles à tout mouvement en faveur d'une direction centralisée. Les promoteurs du projet de fédération régionale eurent passablement de difficultés à réunir le nombre de caisses requis par la loi pour procéder à la fondation de l'Union régionale ${ }^{15}$. Des 19 caisses établies dans le diocèse de Trois-Rivières en 1920, dix acceptèrent de signer la déclaration de fondation; les deux autres signatures étant celles de représentants de caisses fermées ${ }^{16}$. Parmi ce petit groupe de caisses nouvellement fédérées, deux se désaffilièrent dans les deux mois suivant la signature de la déclaration de fondation ${ }^{17}$, ce qui, en 1921 , portait à huit le nombre de caisses affiliées. Une fois l'Union fondée, encore fallait-il la faire reconnaître comme représentante légitime des caisses qui n'avaient pas accepté le nouveau cadre fédéral. Le processus d'affiliation des caisses déjà établies rencontra d'importantes résistances durant les années 1920: «quelques-unes, disait un ancien président régional qui occupait alors un poste d'administrateur, attendirent même d'y être forcées avant de faire le geste nécessaire.» ${ }^{18}$ En effet, l'intervention du Gouvernement du Québec, à travers des dispositions introduites en 1925 et 1930 dans la Loi des syndicats coopératifs, allait finalement leur forcer la main ${ }^{19}$.

15 Un amendement apporté à la Loi des syndicats coopératifs en 1915, permettait en outre à tout groupe composé d'au moins douze caisses de se constituer en fédération.

16 Au nombre des signataires de la déclaration de fondation de l'URTR, se trouvaient notamment les représentants des caisses de Saint-Maurice, paralysée de 1920 à 1924, et de SaintRoch-de-Mékinac, pourtant fermée depuis 1913.

17 Les signataires représentant les caisses de Saint-Prosper et d'Almaville n'avaient pas été autorisés à signer la déclaration de fondation par leurs conseils d'administration respectifs. Dans les deux cas, les signatures furent désapprouvées et les caisses adoptèrent une résolution à l'effet de ne pas s'affilier. Voir URTR, Procès-verbal de la séance du Conseil d'administration tenue le 13 avril 1921 et Ernest Gravel, président de la Caisse de Saint-Prosper, à Guillaume Courtois, secrétaire de l'URTR, 28 février 1921.

18 Sinaï T. Lupien dans La caisse populaire Desjardins, décembre 1935.

19 L'article 6782 de la Loi des syndicats coopératifs sanctionnée le 3 avril 1925 obligeait les caisses à se faire inspecter au moins une fois l'an par un inspecteur rattaché à une fédération diocésaine. Les caisses non affiliées étaient tenues de faire exécuter cette inspection à leurs propres frais. L'amendement de 1930, pour sa part, introduisait le droit de regard et d'inspection gouvernementale à la demande d'un Conseil d'administration, d'un Conseil de surveillance ou encore de 25 membres (ou les deux tiers) en règle. 
Cette opposition à la reconnaissance du nouveau cadre fédéral se prolongea durant une dizaine d'années. L'Union régionale regroupait 20 des 33 caisses en activité à la fin de l'année 1924, soit environ $60 \%$ des effectifs. En 1926, cette proportion passait aux trois quarts et en 1930 l'URTR ralliait la presque totalité des caisses actives, soit 35 sur 36. Nos données indiquent par ailleurs que l'opposition à la reconnaissance du cadre fédéral était principalement localisée du côté sud du Saint-Laurent. Aux prises avec cette persistance des caisses de première souche à ne pas reconnaître la légitimité de son existence, l'URTR prenait l'initiative d'en créer de nouvelles. Poursuivant la stratégie amorcée sous le leadership de Desjardins, les dirigeants régionaux allaient privilégier l'établissement de caisses dans les localités rurales et porter une attention particulière à la consolidation des positions de la jeune Union sur la rive sud du Saint-Laurent. À maintes occasions, ils exprimèrent leur intention d'établir des caisses dans chacune des paroisses: «Une caisse par paroisse», tel est le mot d'ordre que se réapproprient durant les années 1920 les leaders de l'URTR $^{20}$. Cette stratégie d'implantation territoriale était étroitement associée à la question du crédit agricole. Un long débat se déroule pendant les années 1920-1930 sur l'opportunité pour les caisses d'offrir à la classe agricole un crédit à long terme ${ }^{21}$. La progression trop lente du mouvement des caisses, aussi bien en termes de nombre d'établissements qu'en termes de capacité financière, a amené les agriculteurs organisés à se tourner vers l'État provincial. Ce qui donna lieu en 1937 à la création de l'Office du crédit agricole. Les caisses rurales pour leur part ont continué à se cantonner dans le crédit à court terme, à savoir l'achat de graines de semence, d'animaux ou d'instruments aratoires.

À la fin de 1935, plus de 25 ans après l'apparition des premiers établissements dans la région, un total de 74 caisses avaient été organisées, 22 avaient fermé et cinq avaient été réorganisées. Les 57 caisses actives comptaient plus de 9000 sociétaires et géraient un actif total de 1,7 million\$. Vingt-trois d'entre elles n'avaient pas plus de

20 Ils reprenaient en fait le mot d'ordre lancé à plusieurs reprises par les autorités religieuses et notamment par l'Évêché de Trois-Rivières durant les années 1910 . À ce sujet, Union régionale de Trois-Rivières, Procès-verbaux des séances du conseil d'administration tenues les 27 avril 1922 et 31 août 1922; Procès-verbal de l'assemblée générale annuelle tenue le 11 décembre 1923; "Tous pour chacun et chacun pour tous»: lettre circulaire adressée aux curés des diocèses de Trois-Rivières et de Nicolet, avril 1922; Rapport des travaux et délibérations du Congrès des caisses populaires Desjardins tenu à Québec les 6, 7 et 8 octobre 1925.

21 Voir Jean-Pierre Kesteman, Histoire du syndicalisme agricole U.C.C.-U.P.A. 19241984 (Montréal, Boréal, 1984), 75-78; Michel Morissette, L'agriculture familiale au Québec (Paris, L'Harmattan, 1987), 31-38. 
trois années d'existence, résultat des efforts déployés au début des années 1930 pour étendre le mouvement à la rive sud du SaintLaurent ${ }^{22}$. Parmi les caisses actives, 32 sont localisées du côté de la rive sud du Saint-Laurent. Plus nombreuses et plus jeunes, les caisses de la rive sud sont néanmoins de dimension beaucoup plus modeste que les caisses de la rive opposée: elles rassemblent $43 \%$ de l'ensemble des sociétaires mais ne disposent que de $20 \%$ de l'actif total des établissements affiliés. Le mouvement Desjardins reste largement tributaire de ses liens avec le monde rural. Regroupant 6700 sociétaires et cumulant un actif d'un million de dollars, les 51 caisses rurales dominent largement l'Union régionale: elles représentent ainsi plus de $70 \%$ des sociétaires des caisses affiliées et contrôlent plus de $60 \%$ de l'actif du mouvement ${ }^{23}$. Par ailleurs, celui-ci est travaillé par de profondes disparités: en 1935, 70\% de l'actif géré par les 57 caisses affiliées à l'URTR est localisé dans les coffres d'une dizaine d'entre elles. La seule caisse de Trois-Rivières dispose du quart de cet actif.

\section{UN MOUVEMENT ANIMÉ PAR LE MONDE AGRICOLE ET LA PETITE BOURGEOISIE}

Sur quelles forces sociales les caisses se sont-elles appuyées durant cette phase initiale de leur évolution? Deux phénomènes ressortent à l'analyse des données tirées de leurs déclarations de fondation et de la progression de leur sociétariat: le faible enracinement des caisses dans les localités desservies de même que la relative homogénéité des assises sociales du mouvement à l'échelle régionale.

L'adhésion aux caisses et, à plus forte raison, la participation à leur direction, suppose de l'aisance: il faut de l'argent pour acheter sa part sociale, épargner sur son revenu et emprunter; il faut aussi du temps et de l'instruction pour participer à la gestion. Entre les années 1920 et 1935 , le nombre de sociétaires s'accroît de $50 \%$ dans la région; nos données indiquent par ailleurs que cette croissance ne

22 En 1935, toutes les caisses en activité sur le territoire de juridiction de l'URTR sont affiliées. Le sociétariat de l'URTR ne se résume cependant pas à ces seules caisses. En effet, nous avons recensé un total de 26 autres caisses affiliées à l'URTR entre les années 1925 et 1935 qui ne sont pas prises en compte ici et dans l'ensemble de nos données. Ces caisses, souvent établies par des promoteurs de l'URTR, étaient implantées sur les territoires des diocèses de Joliette, Sherbrooke et Saint-Hyacinthe ainsi que dans la région de l'Abitibi. La plupart quitteront les rangs de l'URTR durant les années 1930 et 1940 alors que des Unions régionales verront le jour dans leurs régions d'appartenance. Aux caisses des autres diocèses, il faudrait également ajouter un certain nombre de coopératives agricoles qui, ayant contracté un emprunt de la caisse régionale, lui étaient affiliées.

23 Les données relatives à la situation des caisses au terme de l'année 1935 sont tirées des Rapports mensuels des caisses au 31 décembre 1935. 
procède pas tant d'un enracinement des caisses dans leur milieu que de l'accroissement de leur nombre. À la veille de la Seconde Guerre, une trentaine d'années après leur introduction sur le territoire de l'Union régionale de Trois-Rivières, les caisses ne parviennent toujours pas à rassembler plus de $10 \%$ des résidants des localités desservies et le crédit n'y est accessible qu'à une infime minorité ${ }^{24}$. Dans les meilleurs cas, celui des caisses rurales établies de longue date, le taux d'adhésion des populations locales oscille autour de $15 \%$; dans le cas des caisses urbaines, le taux se situe autour de $5 \%$. La proportion des emprunteurs dans les populations desservies est passablement limitée: $2 \%$ dans le cas des caisses rurales et $0,5 \%$ dans celui des caisses urbaines entre 1915 et 1935 . Partant de l'examen de cinq cas types de caisses, les travaux de Ronald Rudin ont bien démontré à cet égard que, jusqu'à la Seconde Guerre du moins, ce sont les éléments plutôt prospères des localités d'implantation des caisses qui adhèrent au mouvement ${ }^{25}$. Nous ne disposons pas des informations qui nous permettent de corroborer cette hypothèse; une telle entreprise à l'échelle de l'ensemble des caisses exigerait un investissement démesuré. Plusieurs éléments peuvent expliquer, en partie, le caractère ténu des rapports entre les caisses et les populations paroissiales durant cette première phase de leur évolution. Il faut d'abord souligner que les conditions d'existence de larges segments des classes agricole et ouvrière demeurent passablement difficiles pendant l'entre-deuxguerres. L'intérêt, voire la capacité, d'adhérer aux caisses s'en trouvent amoindris. En effet, l'acquisition de la part sociale et le paiement de la «taxe d'entrée» continuent à représenter une dépense non négligeable pour bon nombre d'ouvriers et d'agriculteurs ${ }^{26}$.

24 Les taux d'adhésion ont été calculés en mettant en rapport le sociétariat de chacune des caisses et la population de leurs circonscriptions territoriales respectives. Les populations des localités non desservies ne sont pas prises en compte; ce qui donne une image plus précise des rapports entre les caisses et leur milieu ambiant. Les sources et les ouvrages suivants ont été mis à contribution: Jean Roy, D. Robert et Louise Verreault-Roy, Les populations municipales et paroissiales de la Mauricie. Dossier statistique: 1850-1971 (Trois-Rivières, Publication du Groupe de recherche sur la Mauricie, UQTR, 1980); Statistique Canada, Recensements, années 1931, 1941, 1951, 1961; Le Canada ecclésiastique (Montréal, Librairie Beauchemin Limitée, éditions 1916-1966).

25 Ronald Rudin, In Whose Interest? Quebec's Caisses populaires, 1900-1945 (Montréal et Kingston, McGill-Queen's University Press, 1990), 28-44.

26 Rudin a suggéré certains éléments d'interprétation de la faiblesse des taux d'adhésion. Il a souligné, à partir du cas de la Caisse de Lévis, que le relèvement de la «taxe d'entrée» pouvait constituer un obstacle au recrutement de nouveaux membres. Cette politique, poursuivie par les dirigeants de la caisse, aurait entraîné une contraction du sociétariat au profit d'une augmentation du nombre de déposants. Ibidem, 42-44. Ces pratiques ne semblent cependant pas avoir été généralisées dans la région de la Mauricie et des Bois-Francs. 
TABLEAU 1

Les fondateurs de caisses populaires selon la catégorie socioprofessionnelle et le statut au terme de l'assemblée de fondation

Union régionale de Trois-Rivières, 1909-1935*

nombres absolus et (\%)

\begin{tabular}{|c|c|c|c|c|c|c|}
\hline & & $\begin{array}{l}\text { blissements } \\
\text { urbains }\end{array}$ & & & $\begin{array}{l}\text { ablissement } \\
\text { ruraux }\end{array}$ & \\
\hline catégories / statuts & non élus & élus & total & non élus & élus & total \\
\hline 1. commerçants, entrep. \& industriels & $13(10,4)$ & $11(22,9)$ & $24(13,9)$ & $29(4,4)$ & $28(10,1)$ & $57(6,3)$ \\
\hline 2. cadres et administrateurs & $6(4,8)$ & $3(6,3)$ & $9(5,2)$ & $3(0,5)$ & $4(1,4)$ & $7(0,8)$ \\
\hline 3. membres des professions libérales & $3(2,4)$ & $14(29,2)$ & $17(9,8)$ & $19(2,9)$ & $45(16,3)$ & $64(6,9)$ \\
\hline 4. spécialistes et techniciens & $1(0,8)$ & $2(4,2)$ & $3(1,7)$ & $8(1,2)$ & $2(0,7)$ & $10(1,2)$ \\
\hline 5. employés & $4(3,2)$ & $1(2,1)$ & $5(2,9)$ & $6(0,9)$ & $1(0,4)$ & $7(0,8)$ \\
\hline 6. cultivateurs et professions assimilées & $48(38,4)$ & $7(14,0)$ & $55(31,8)$ & $489(74,7)$ & $170(61,6)$ & $659(70,9)$ \\
\hline 7. artisans et gens de métiers & $21(16,8)$ & $4(8,3)$ & $25(14,4)$ & $45(6,9)$ & $14(5,1)$ & $59(6,3)$ \\
\hline - artisans & $17(13,6)$ & $3(6,3)$ & $20(11,6)$ & $34(5,2)$ & $11(4,0)$ & $45(4,8)$ \\
\hline - gens de métiers & $4(3,2)$ & $1(2,1)$ & $5(2,9)$ & $11(1,7)$ & $3(1,1)$ & $14(1,5)$ \\
\hline 8. ouvriers & $24(19,2)$ & $4(8,3)$ & $28(16,2)$ & $39(6,0)$ & $4(1,4)$ & $43(4,6)$ \\
\hline - contremaitres & $2(1,6)$ & $3(6,3)$ & $5(2,9)$ & $0(0,0)$ & $0(0,0)$ & $0(0,0)$ \\
\hline - spécialisés et non spécialisés & $22(17,6)$ & $1(2,1)$ & $23(13,3)$ & $39(6,6)$ & $4(1,4)$ & $43(4,6)$ \\
\hline 9. rentiers & $5(4,0)$ & $2(4,2)$ & $7(4,1)$ & $17(2,6)$ & $8(2,9)$ & $25(2,7)$ \\
\hline TOTAUX & $125(100,0)$ & $48(100,0)$ & $173(100,0)$ & $655(100,0)$ & $276(100,0)$ & $931(100,0)$ \\
\hline
\end{tabular}

Sources: Déclarations de fondation, procès-verbaux des assemblées de fondation et des premières séances des conseils d'administration des caisses populaires affiliées à l'URTR.

* Les professions indéterminées ainsi que les sociétaires auxiliaires ont été éliminés du classement. 
Quels groupes sociaux se sont mobilisés pour fonder les caisses et pour assurer leur fonctionnement? Le tableau 1 a été reconstitué à partir d'une recension exhaustive des participants aux assemblées de fondation de chacune des caisses affiliées à l'URTR, de leur profession et de leur statut au sein de l'organisation au terme de leur élection à l'un ou l'autre des trois conseils. Nous retiendrons trois faits marquants dans le cas des établissements fondés entre 1909 et 1935: le poids considérable des cultivateurs parmi les fondateurs, la présence pour le moins discrète du monde des employés et des ouvriers ainsi que la représentation significative des éléments associés à la petite propriété (artisans, commerçants et industriels locaux, etc.) et aux membres des professions libérales (incluant le clergé).

Sept fondateurs de caisses sur dix se déclarent cultivateurs dans les localités rurales. Ils y dominent par ailleurs largement les conseils d'administration et les commissions de crédit, de même qu'ils forment la moitié des effectifs des conseils de surveillance. Comptant pour $20 \%$ des signataires des déclarations de fondation, les gens d'affaires locaux (marchands, entrepreneurs et artisans) et les représentants des professions libérales réunis fournissent le tiers des administrateurs, le cinquième des commissaires de crédit et près de la moitié des conseillers de surveillance des caisses rurales. À l'inverse, le poids du monde ouvrier, des employés, du personnel cadre et spécialisé est quasi négligeable tant au niveau des participants aux assemblées de fondation qu'à celui des élus.

On devrait s'attendre à une meilleure représentation des ouvriers et des employés dans le cas des caisses urbaines. Malgré une meilleure visibilité, ils demeurent peu représentés, comptant pour à peine $20 \%$ des fondateurs et $10 \%$ des élus. À ces chapitres, ils sont nettement surpassés par les cultivateurs. En revanche, la nébuleuse des gens d'affaires (artisans, commerçants et entrepreneurs) fournit un bon contingent des fondateurs et des élus: pas moins de trois fondateurs sur dix et le tiers des élus aux trois conseils appartiennent à cet ensemble composite. Les membres des professions libérales et du clergé, pour leur part, représentent un peu moins de $10 \%$ des participants aux assemblées de fondation. Ils siègent cependant à l'un ou l'autre des trois conseils dans une proportion de $30 \%$.

Alphonse Desjardins, nous l'avons vu précédemment, misait beaucoup sur la mobilisation du clergé paroissial pour multiplier les caisses. Comment cette participation s'est-elle effectivement traduite dans la région? Mentionnons d'abord que son poids relatif n'est pas tant fonction du nombre que de l'influence exercée à la direction des établissements. Des 54 curés ou vicaires engagés dans les fondations des 49 caisses pour lesquelles nous disposons de données pendant 
cette période, 36 siègent à l'un ou l'autre des trois conseils. Par ailleurs, bien que leur participation nominale soit constante pour l'ensemble de la période (environ un prêtre par fondation), ils sont beaucoup plus actifs du vivant d'Alphonse Desjardins alors qu'ils occupent les postes clés de l'organisation locale; durant les années postérieures à son décès, ils jouent un rôle plus effacé ${ }^{27}$.

Replacées dans leurs contextes respectifs, ces alliances localisées entre petits producteurs agricoles, marchands, membres des professions libérales et représentants du clergé mettent en jeu des intérêts le plus souvent associés à ceux des figures dominantes de la paroisse. En effet, les notables sont généralement des intermédiaires privilégiés pour y introduire les caisses; ils se solidarisent avec leur milieu social selon des scénarios diversifiés. Ainsi à la Caisse de Saint-Étienne-desGrès, fondée en 1912 par A. Desjardins, dans une paroisse à vocation agricole de 1500 habitants, 64 participants souscrivent un total de 286 parts sociales. Au terme de l'assemblée de fondation, la présidence de la caisse est confiée au curé de la paroisse et la gérance à un homme d'affaires du lieu. Un médecin, un autre homme d'affaires, deux artisans, trois cultivateurs, un marchand et un journalier remplissent les autres charges. Fait significatif, les onze élus détiennent près de $60 \%$ des parts sociales émises au cours de cette assemblée de fondation. Parmi les 42 membres dont la profession est connue, 18 sont cultivateurs ${ }^{28}$. L'assemblée de fondation de la Caisse de TroisRivières, pour sa part, a lieu en 1909 en présence d'Alphonse Desjardins. Elle mobilise une quinzaine de notables très en vue de la localité. Un curé, un médecin, alors maire de la ville, et un notaire en assument respectivement la présidence, la vice-présidence et la gérance. Ils sont entourés d'une équipe composée d'un autre représentant de la profession médicale, de deux avocats, de quatre marchands, d'un entrepreneur et de quatre individus associés au monde des affaires trifluvien ${ }^{29}$. La fondation en 1935 de la Caisse de Tingwick, une localité rurale de 1500 habitants située au sud de Victoriaville, offre par ailleurs un autre scénario. Avec 83 des 103

27 Parmi les 21 curés ou vicaires signataires des déclarations de fondation des 18 établissements mis sur pied entre 1909 et 1920 et pour lesquels les informations sont disponibles, 14 sont élus à leurs conseils d'administration (dont dix à titre de président et trois à celui de gérant) et deux le sont à leurs conseils de surveillance. Entre 1921 et 1935, les 33 prêtres recensés dans les déclarations de fondation disponibles ne sont plus que cinq à exercer une charge aux conseils d'administration locaux; ils sont cependant 15 à siéger aux conseils de surveillance.

28 Caisse de Saint-Étienne-des-Grès, Déclaration de fondation, Procès-verbal de l'Assemblée générale, Procès-verbal du Conseil d'administration, 6 juin 1912.

29 Caisse de Trois-Rivières, Déclaration de fondation, Procès-verbal de l'Assemblée générale, Procès-verbal du Conseil d'administration, 24 octobre 1909. 
participants, les agriculteurs dominent largement l'assemblée. Au terme des élections, ils détiennent dix des 14 sièges aux trois conseils, les autres étant occupés par le vicaire, un médecin, un menuisier et un industriel de l'endroit ${ }^{30}$.

Tant en milieu urbain que rural, une constante apparaît à l'examen des fondateurs de caisses et de leurs mandataires durant la phase initiale de leur implantation: ces fondations ont été l'occasion pour les agents les plus dynamiques de la petite propriété de se solidariser avec les éléments des professions libérales et le clergé paroissial. Particulièrement actifs pendant les années 1910, les membres du clergé ont joué par la suite un rôle plus discret. L'implantation initiale des caisses s'est réalisée principalement en milieu rural pour plusieurs raisons. Desjardins associait étroitement le développement des caisses à celui de l'agriculture, à l'accroissement de sa productivité par l'utilisation de méthodes modernes de culture et de mise en marché ${ }^{31}$. Pour l'Église, comme pour Desjardins, l'agriculture demeure l'activité économique privilégiée. De façon générale, les bouleversements économiques et sociaux que connaît le monde rural favorisent le déploiement d'initiatives convergentes avec le projet de Desjardins. Mentionnons à titre indicatif l'essor des premières coopératives agricoles au tournant des années 1910 et la naissance des premiers syndicats d'agriculteurs une dizaine d'années plus tard ${ }^{32}$. La question du crédit agricole sera au cœur des revendications portées par les agriculteurs organisés pendant l'entre-deux-guerres. Elle est directement associée à la stratégie d'implantation des caisses populaires préconisée par les successeurs de Desjardins jusqu'au milieu des années 1930. Il faut enfin souligner que les banques privilégient à cette époque les milieux urbains, laissant les ruraux sans institution financière, à la merci du prêt usuraire, et les caisses sans concurrents ${ }^{33}$. En définitive, l'émergence des caisses pendant ce premier tiers du siècle témoigne à plusieurs égards d'une résistance des éléments anciens de la petite bourgeoisie et du monde agricole à la précarisation de leurs positions dans le contexte du déclin relatif de la petite production locale. Elle

30 Caisse de Tingwick, Déclaration de fondation, Procès-verbal de l'Assemblée générale, Procès-verbal du Conseil d'administration, 4 mars 1935.

31 À ce sujet, on peut se référer à la section que Desjardins intitule «Le rôle de la caisse populaire dans l'agriculture et la colonisation», dans le «Mémoire sur l'organisation de l'agriculture dans la province de Québec», op. cit., 178-184.

32 À ce sujet Claude Beauchamp, «Les débuts de la coopération et du syndicalisme agricoles, 1900-1930: quelques éléments de la pratique», Recherches sociographiques, 20,3 (sept.-déc. 1979): 337-381.

33 Ronald Rudin, Banking en français. Les banques canadiennes-françaises 1835-1925 (Montréal, Boréal, 1985). 
traduit, d'autre part, la volonté d'adaptation de leurs éléments les plus novateurs à l'économie de marché.

\section{2 - L'ENRACINEMENT DU MOUVEMENT (1935-1945)}

À compter du milieu des années 1930, l'évolution des caisses allait connaître une nouvelle tournure. La création de la Fédération de Québec des Unions régionales en 1932, l'octroi d'une subvention gouvernementale annuelle pour l'établissement et l'inspection des caisses populaires ainsi qu'une croissance économique nourrie par l'effort de guerre vont créer un climat particulièrement favorable au décollage du mouvement des caisses. En l'espace de dix ans, celles-ci vont littéralement quadriller le territoire.

\section{LA PROPAGATION DES CAISSES}

Les années qui vont du milieu des années 1930 à la fin de la guerre se caractérisent par la poursuite des efforts intensifs pour étendre le réseau des caisses, à la différence toutefois que des énergies vont être engagées pour renforcer ses positions dans les villes. Au terme de la guerre, l'objectif d'établir «une caisse par paroisse» est partiellement réalisé: 129 des 144 paroisses comprises dans l'aire d'activité de l'Union ont leurs propres établissements.

Pas moins de 16 réorganisations et 57 fondations ont lieu à l'échelle de la région de 1936 à 1945, soit plus de la moitié des établissements en activité à la fin de la guerre. Une seule caisse cessera ses opérations durant ces années alors que, dans la période antérieure, les établissements nouvellement fondés avaient à peine compensé les fermetures. Les caisses réalisent par ailleurs des percées significatives dans les villes où leur nombre est porté de 6 à 32 .

La conjoncture qui prévaut durant la guerre est tout à fait exceptionnelle. La croissance économique accélérée et les restrictions à la consommation imposées par l'économie de guerre favorisent l'épargne. Les caisses, particulièrement dans les villes, atteignent des taux de croissance et un rythme de développement inégalés. En l'espace de cinq ans, l'actif de l'ensemble des caisses affiliées est multiplié par six et leur sociétariat par trois. L'actif des caisses urbaines est multiplié par vingt et le nombre de leurs sociétaires quadruple alors que les caisses rurales voient leur actif quintupler et le nombre de leurs sociétaires doubler.

Au sortir de la guerre, le mouvement Desjardins est profondément modifié. Après s'être longuement appuyé sur le poids des ruraux, il s'ouvre résolument à la réalité urbaine. Les caisses rurales rassemblaient encore les deux tiers des sociétaires du mouvement en 1940, 
TABLEAU 2

Progression des caisses populaires selon le nombre d'établissements, le nombre de sociétaires et l'actif

Union régionale de Trois-Rivières, 1915-1965*

\begin{tabular}{|c|c|c|c|c|c|c|}
\hline amnées & $\begin{array}{c}\text { caisses } \\
\text { actives (na) }\end{array}$ & $\begin{array}{l}\text { sociétaires } \\
\text { (na.) }\end{array}$ & actif $(000 \$)$ & $\begin{array}{c}\text { caisses } \\
\text { actives (n.a) }\end{array}$ & $\begin{array}{c}\text { sociétaires } \\
\text { (na) }\end{array}$ & actif $(000 \$)$ \\
\hline 1915 & 20 & 3524 & 230 & 2 & 886 & 73 \\
\hline 1920 & 25 & 3995 & 644 & 4 & 1523 & 345 \\
\hline 1925 & 28 & 4361 & 725 & 6 & 1823 & 490 \\
\hline 1930 & 29 & 6154 & 1054 & 7 & 2717 & 770 \\
\hline 1935 & 51 & 6691 & 1084 & 6 & 2658 & 676 \\
\hline 1940 & 74 & 13062 & 2214 & 18 & 6831 & 1421 \\
\hline 1945 & 97 & 28689 & 11344 & 32 & 28735 & 10321 \\
\hline 1950 & 101 & 38110 & 16841 & 34 & 50262 & 22593 \\
\hline 1955 & 98 & 44572 & 21261 & 37 & 70975 & 37523 \\
\hline 1960 & 98 & 54247 & 29447 & 40 & 96318 & 63416 \\
\hline 1965 & 97 & 66899 & 46500 & 42 & 135759 & 102818 \\
\hline
\end{tabular}

* Sources: Annuaires du Québec, 1915-1933; Rapports mensuels des caisses au 31 décembre, 1935-1965, Procèsverbaux de l'Union régionale de Trois-Rivières, 1920-1965; Déclarations de fondation des caisses populaires. 
et disposaient toujours de $60 \%$ de son actif. Cinq ans plus tard, elles se partagent à peu près également les effectifs du mouvement avec les caisses urbaines tant sur le plan du sociétariat que de l'actif, bien que les dernières soient beaucoup moins nombreuses et généralement plus jeunes. L'expansion des années 1935-1945 favorise une meilleure répartition régionale des effectifs du mouvement. Avec 72 des 129 établissements en activité à la fin de l'année 1945, les caisses de la rive sud voient leur poids financier gagner en importance par rapport à celui des caisses de la rive opposée. Leur part de l'actif régional passe en effet de 20 à $40 \%$ entre 1935 et 1945 . Une dizaine d'années aura suffi à l'URTR pour étendre son réseau d'établissements à l'ensemble du territoire alors qu'elle avait eu peine à maintenir ses positions durant la période antérieure.

\section{L'OUVERTURE SUR LA VILLE}

La croissance du sociétariat depuis les origines s'était réalisée à l'horizontale, suivant le rythme imprimé par l'accroissement du nombre d'établissements et recrutant l'essentiel de ses effectifs parmi les éléments du monde agricole et de la petite bourgeoisie locale. Ce faisant, le mouvement Desjardins était resté peu sensible à l'urbanisation et à l'industrialisation régionales ainsi qu'aux nouvelles classes salariées dont les effectifs devenaient de plus en plus imposants. Les percées dans les villes à compter de la seconde moitié des années 1930 vont modifier de façon substantielle ses bases sociales. Bien qu'encore abondamment représentés, les agriculteurs et les éléments anciens de la petite bourgeoisie vont désormais composer avec des groupes nouvellement mobilisés au sein des caisses urbaines qui connaissent alors une croissance accélérée.

À compter de 1940, les caisses Desjardins recrutent plus largement parmi les populations desservies qu'elles ne le faisaient jadis. L'adhésion aux caisses s'étend à des segments nettement plus importants des populations locales: en 1945, un résidant sur cinq était membre de la caisse populaire de sa localité. On se souviendra que cette proportion n'atteignait pas $10 \%$ en 1940 . Les caisses rurales recueillaient l'adhésion de $28 \%$ des habitants de leur localité en 1945 alors que les caisses urbaines en obtenaient $16 \%$ la même année. Ces proportions étaient respectivement de 17 et de 5\% cinq ans plus tôt.

Cet enracinement se confirme à l'examen de la composition sociale des fondateurs et des élus lors d'assemblées de fondation de caisses. Ce sont d'abord les caisses urbaines qui vont ouvrir cette première brèche. En effet, la composition des assemblées de fondation dans le cas des caisses rurales est à peu près similaire à ce qu'elle était durant la période antérieure. Elles continuent à mobiliser très 
TABLEAU 3

Les fondateurs de caisses populaires selon la catégorie socioprofessionnelle

et le statut au terme de l'assemblée de fondation

Union régionale de Trois-Rivières, 1936-1945*

nombres absolus et (\%)

établissements

établissements. urbains

\begin{tabular}{|l|rrrrrr|}
\hline \multicolumn{1}{|c}{ catégories / statuts } & \multicolumn{1}{c}{ non élus } & \multicolumn{1}{c}{ ćlus } & \multicolumn{1}{c|}{ total } & \multicolumn{1}{c|}{ non élus } & \multicolumn{1}{c|}{ élus } & \multicolumn{1}{c|}{ total } \\
\hline 1. commerçants, entrep. \& industriels & $63(10,8)$ & $44(18,3)$ & $107(13,0)$ & $65(6,1)$ & $43(8,5)$ & $108(6,9)$ \\
2. cadres et administrateurs & $7(1,2)$ & $8(3,3)$ & $15(1,8)$ & $9(0,9)$ & $7(1,4)$ & $16(1,0)$ \\
3. membres des professions libérales & $34(5,8)$ & $14(5,8)$ & $48(5,8)$ & $36(3,4)$ & $36(7,1)$ & $72(4,0)$ \\
4. spécialistes et techniciens & $10(1,7)$ & $14(5,8)$ & $24(2,9)$ & $17(1,6)$ & $9(1,8)$ & $26(1,7)$ \\
5. employés & $44(7,6)$ & $20(8,3)$ & $64(7,8)$ & $14(1,3)$ & $4(0,8)$ & $18(1,2)$ \\
6. cultivateurs et professions assimilceses & $104(17,8)$ & $41(17,0)$ & $145(17,6)$ & $744(70,3)$ & $341(67,4)$ & $1,085(69,2)$ \\
7. artisans et gens de métiers & $96(16,5)$ & $37(15,4)$ & $133(16,1)$ & $75(7,1)$ & $36(7,1)$ & $111(7,1)$ \\
- artisans & $27(4,6)$ & $9(3,7)$ & $36(4,4)$ & $51(4,8)$ & $25(4,9)$ & $76(4,9)$ \\
- gens de métiers & $69(11,8)$ & $28(11,6)$ & $97(11,8)$ & $24(2,3)$ & $11(2,2)$ & $35(2,2)$ \\
8. ouvriers & $212(36,4)$ & $59(24,5)$ & $271(32,9)$ & $79(7,5)$ & $18(3,6)$ & $97(6,2)$ \\
- contremaîtres & $17(2,9)$ & $14(5,8)$ & $31(3,8)$ & $3(0,3)$ & $2(0,4)$ & $5(0,3)$ \\
- spécialisés et non spécialisés & $195(33,6)$ & $45(18,7)$ & $240(29,1)$ & $76(7,9)$ & $16(3,2)$ & $92(5,9)$ \\
9. rentiers & $13(2,2)$ & $4(1,7)$ & $17(2,1)$ & $19(1,8)$ & $11(2,2)$ & $30(1,9)$ \\
TOTAUX & $583(100,0)$ & $241(100,0)$ & $824(100,0)$ & $1,058(100,0)$ & $506(100,0)$ & $1,564(100,0)$ \\
\hline
\end{tabular}

Sources: Déclarations de fondation, procès-verbaux des assemblées de fondation et des premières séances des conseils d'administration des caisses populaires affiliées à l'URTR.

* Les professions indéterminées ainsi que les sociétaires auxiliaires ont été éliminés du classement. 
largement les agriculteurs qui représentent plus des deux tiers des participants aux assemblées de fondation et dominent nettement leurs trois conseils. Les assemblées des caisses urbaines reflètent davantage qu'auparavant la diversité de leurs milieux respectifs (tableau 3). Bien qu'encore amplement représentés aux postes clés, les agents de la petite propriété et les professions libérales doivent transiger avec l'arrivée de nouveaux groupes. Cette diversification des intérêts en présence se traduit par les percées réalisées parmi les salariés (personnel cadre et spécialisé, employés et ouvriers réunis). Ils forment près de la moitié des participants aux assemblées de fondation des caisses urbaines érigées entre 1936 et 1945 et détiennent quatre sièges sur dix aux trois conseils. Ils ne représentaient que le quart des fondateurs et moins du cinquième des élus durant la période précédente.

Par ailleurs, les assemblées de fondation dans les paroisses urbaines s'ouvrent à des acteurs aux intérêts plus diversifiés que dans les situations observées précédemment. Ainsi, la Caisse de SaintFrançois-d'Assise, établie dans une paroisse ouvrière de TroisRivières en 1944, est fondée au cours d'une assemblée réunissant 48 participants. Trois des 19 ouvriers participant à l'assemblée sont élus à l'un ou l'autre des trois conseils de la caisse; les neuf commerçants présents y occupent, pour leur part, cinq des onze sièges ${ }^{34}$. Une assemblée composée à $70 \%$ d'ouvriers donne naissance à la Caisse de Saint-Simon en 1936, dans une paroisse de Drummondville peuplée de travailleurs du textile. Au terme de la tenue des élections, les ouvriers sont titulaires de quatre sièges dont trois au conseil de surveillance. Les postes clés restent cependant du ressort de la petite bourgeoisie locale: la gérance est confiée à un notaire et la présidence du conseil d'administration à un rentier. La commission de crédit est présidée par un propriétaire foncier, les deux autres charges étant confiées à des marchands ${ }^{35}$.

La période 1935-1945 correspond également à un nouveau recul des positions du clergé à la direction des caisses. Les curés continuent à s'associer à la cause des caisses Desjardins en participant aux assemblées de fondation mais ils le font davantage à titre de sociétaires et sont de moins en moins nombreux à figurer parmi les élus. Lorsqu'ils sont élus, ils siègent aux conseils de surveillance, qui pour la plupart exercent un rôle négligeable dans l'administration des caisses en dépit des prescriptions de la Loi des syndicats coopératifs. Des 70 curés ou

34 Caisse de Saint-François-d'Assise, Déclaration de fondation, Procès-verbal de l'Assemblée générale, Procès-verbal du Conseil d'administration, 22 mars 1944.

35 Caisse de Saint-Simon, Déclaration de fondation, Procès-verbal de l'Assemblée générale, Procès-verbal du Conseil d'administration, 14 décembre 1936. 
vicaires recensés dans les déclarations de fondations des caisses établies entre 1936 et 1945, seulement trois détiennent un siège d'administrateur alors que 19 sont élus aux conseils de surveillance.

En somme, cette période de croissance est celle d'une conquête de la région menée sur trois fronts: un quadrillage du territoire par l'établissement d'une caisse par paroisse; un recrutement plus intensif parmi les populations locales; et enfin, une percée significative dans les villes. La diffusion des caisses en milieu urbain se réalisa au rythme du peuplement des nouvelles paroisses érigées par l'Église. Dans l'esprit des leaders du mouvement, la paroisse urbaine offrait aux caisses un tissu social apparenté à l'image qu'ils avaient de la communauté rurale: des communautés stables, solidaires de leurs notables, attachées aux valeurs religieuses et familiales et soucieuses d'établir leurs enfants. Elle offrait aussi un cadre suffisamment restreint pour que puissent jouer les relations d'inter-connaissance dans l'octroi du crédit et la protection de l'épargne. Assez rapidement, comme nous le verrons plus loin, la mutualité paroissiale va se frotter à la réalité du marché en milieu urbain: le voisinage entre caisses inégalement développées et la concurrence avec les autres institutions financières sont au nombre des enjeux qui pointent au sortir de la guerre.

\section{3 - UNE NOUVELLE DONNE DANS L'APRÈS-GUERRE (1945-1965)}

La période qui s'achève avec la fin de la guerre aura été l'occasion pour le mouvement des caisses d'entreprendre le virage imposé par la croissance économique et démographique du front urbain. La suivante voit cette coloration urbaine nouvellement acquise déteindre de plus en plus fortement sur sa dynamique de croissance. Globalement, ces années qui vont de l'après-guerre à la Révolution tranquille sont marquées par un renforcement des tendances antérieurement apparues. Elles vont se traduire par une transformation des principales caractéristiques des bases sociales de son organisation.

Le Québec, comme l'ensemble de l'Occident en général, connaît alors une forte croissance économique stimulée par un accroissement substantiel des investissements et de la production. La migration des ruraux vers les villes, temporairement stoppée pendant la crise et la guerre, reprend à un rythme accéléré. Au Québec, entre 1951 et 1961, la part relative de la population agricole enregistre une chute importante, passant de 20 à $11 \%{ }^{36}$. Par ailleurs, le niveau de vie des

36 Paul-André Linteau, René Durocher, Jean-Claude Robert et François Ricard, Histoire du Québec contemporain, tome 2: Le Québec depuis 1930 (Montréal, Boréal, 1986), 188. 
Québécois n'a rien de commun avec ce qu'ils avaient connu durant les années trente. Le revenu personnel passe en effet de $655 \$$ en 1946 à $1455 \$$ en 1961 , soit un rythme d'accroissement nettement supérieur à celui de l'inflation ${ }^{37}$.

La croissance générale du mouvement à compter de 1945 est favorisée par cette prospérité relative. L'actif des caisses affiliées à l'URTR est multiplié par sept entre les années 1945 et 1965, passant de 22 à 150 millions de dollars ${ }^{38}$. L'arrivée en masse de nouveaux adhérents vient grossir les rangs du sociétariat des caisses. Elles s'ouvrent à de larges segments de la population régionale. Elles comptent un peu plus de 200000 sociétaires en 1965 comparativement à 57000 en $1945^{39}$. L'accroissement soutenu du pouvoir d'achat et l'expansion que connaissent les villes se traduisent par un recours de plus en plus important au crédit, d'abord hypothécaire puis à la consommation depuis la fin des années 1950. Par ailleurs, cette croissance trahit de profondes inégalités de développement entre les caisses affiliées.

\section{LA PROGRESSION ET LA DIVERSIFICATION DU SOCIÉTARIAT}

La progression du sociétariat durant l'après-guerre se caractérise par la stabilisation du nombre d'établissements, le recrutement intensif de nouveaux sociétaires et la diversification de leurs assises sociales.

En termes de gains nets, peu de nouvelles caisses viennent grossir les rangs de l'Union régionale entre 1945 et 1965, si ce n'est l'ajout d'une dizaine dans des paroisses urbaines nouvellement érigées. Les paroisses rurales de la région accueillent sept nouvelles caisses durant les cinq années qui suivent la guerre; elles sont cependant le théâtre d'un nombre égal de fermetures jusqu'en 1965. En somme, le mouvement dans son ensemble gagne peu en étendue.

C'est en profondeur que les caisses réalisent leurs gains. L'examen de l'évolution du taux d'adhésion dans les localités desservies par les caisses révèle en effet que le recrutement de leurs sociétaires se réalise de plus en plus largement. À l'échelle régionale, le taux d'adhésion aux caisses dans leurs milieux d'implantation passe de un sociétaire pour cinq résidants en 1945 à un pour deux en 1965. En

37 Ibidem.

38 Chiffres obtenus à partir d'un traitement des données tirées des Rapports mensuels des caisses au 31 décembre, années 1945 et 1965.

39 Rapports mensuels des caisses au 31 décembre, années 1945 et 1965. À l'échelle du Québec, les mêmes tendances s'observent: le sociétariat des caisses passe de 371000 à 1759000 membres, leur actif est porté de 120 à 1207 millions de dollars pour les mêmes années. Voir La Revue Desjardins, 34,3 (mars 1968): 51. 
FIGURE I

Adhésion des populations locales aux caisses populaires

Union régionale de Trois-Rivières, 1915-1965*

(nombres absolus)

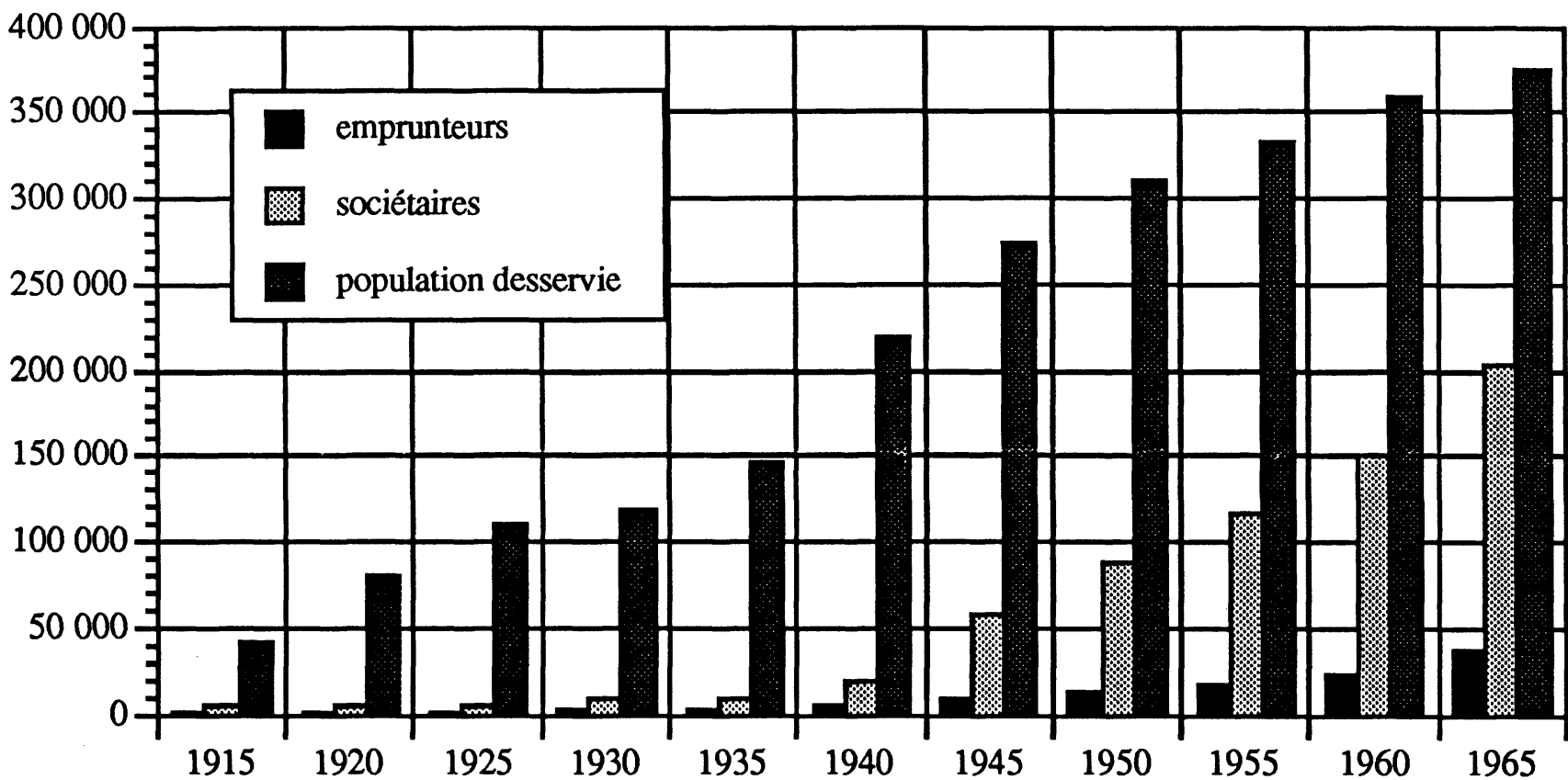

*Sources: Annuaires du Québec, 1915-1930; Rapports mensuels des caisses au 31 décembre, 1935-1965; Le Canada Ecclésiastique, 1915-1965; Recensements fédéraux, 1931, 1941, 1951, 1961; J. Roy et al., Les populations municipales et paroissiales de la Mauricie. Dossier statistique: 1850-1971, Trois-Rivières, Publication du Groupe de recherche sur la Mauricie, cahier no 3, U.Q.T.R., 1980. 
TABLEAU 4

Les fondateurs de caisses populaires selon la catégorie socioprofessionnelle et le statut au terme de l'assemblée de fondation

Union régionale de Trois-Rivières, 1946-1965*

nombres absolus et (\%)

\begin{tabular}{|c|c|c|c|c|c|c|}
\hline \multirow[b]{2}{*}{ catégories / statuts } & \multicolumn{2}{|c|}{$\begin{array}{l}\text { établissements } \\
\text { urbains }\end{array}$} & & \multicolumn{3}{|c|}{$\begin{array}{l}\text { établissements } \\
\text { ruraux }\end{array}$} \\
\hline & non élus & élus & total & non élus & élus & total \\
\hline 1. commerçants, entrep. \& industriels & $44(9,2)$ & $18(13,4)$ & $62(10,1)$ & $11(6,3)$ & $5(8,2)$ & $16(6,8)$ \\
\hline 2. cadres et administrateurs & $47(9,8)$ & $11(8,2)$ & $58(9,4)$ & $5(2,9)$ & $2(3,3)$ & $7(3,0)$ \\
\hline 3. membres des professions libérales & $38(7,9)$ & $14(10,4)$ & $52(8,5)$ & $5(2,9)$ & $4(6,6)$ & $9(3,8)$ \\
\hline 4. spécialistes et techniciens & $54(11,2)$ & $28(20,9)$ & $82(13,3)$ & $4(2,3)$ & $2(3,3)$ & $6(2,5)$ \\
\hline 5. employés & $62(12,9)$ & $11(8,2)$ & $73(11,9)$ & $4(2,3)$ & $3(4,9)$ & $7(3,0)$ \\
\hline 6. cultivateurs et professions assimilées & $6(1,3)$ & $1(0,7)$ & $7(1,1)$ & $92(52,6)$ & $26(42,6)$ & $118(50,0)$ \\
\hline 7. artisans et gens de métiers & $59(12,3)$ & $16(12,0)$ & $75(12,2)$ & $21(12,0)$ & $9(14,8)$ & $30(12,7)$ \\
\hline - artisans & $17(3,5)$ & $6(4,5)$ & $23(3,7)$ & $14(8,0)$ & $6(9,8)$ & $20(8,5)$ \\
\hline - gens de métiers & $42(8,7)$ & $10(7,5)$ & $52(8,5)$ & $7(4,0)$ & $3(4,9)$ & $10(4,2)$ \\
\hline 8. ouvriers & $155(32,2)$ & $33(24,6)$ & $188(30,6)$ & $32(18,3)$ & $9(14,8)$ & $41(17,4)$ \\
\hline - contremaîtres & $15(3,1)$ & $6(4,5)$ & $21(3,4)$ & $2(1,1)$ & $1(1,6)$ & $3(1,3)$ \\
\hline - spécialisés et non spécialisés & $140(29,1)$ & $27(20,1)$ & $167(27,2)$ & $30(17,1)$ & $8(13,1)$ & $38(16,1)$ \\
\hline 9. rentiers & $16(3,3)$ & $2(1,5)$ & $18(2,9)$ & $1(0,6)$ & $0(0,0)$ & $1(0,4)$ \\
\hline TOTAUX & $481(100,0)$ & $134(100,0)$ & $615(100,0)$ & $175(100,0)$ & $61(100,0)$ & $236(100,0)$ \\
\hline
\end{tabular}

Sources: Déclarations de fondation, procès-verbaux des assemblées de fondation et des premières séances des conseils d'administration des caisses populaires affiliées à l'URTR.

* Les professions indéterminées ainsi que les sociétaires auxiliaires ont été éliminés du classement. 
milieu urbain, ces proportions sont respectivement de 17 et $52 \%$ alors qu'elles sont de 28 et $58 \%$ en milieu rural ${ }^{40}$. Cette évolution positive du taux d'adhésion témoigne d'un enracinement des caisses et d'une plus grande diversité du sociétariat. À égalité avec les caisses urbaines vers la fin de la guerre, les caisses rurales voient leur poids relatif s'amenuiser au profit de ces dernières dans les années qui suivent: deux sociétaires sur trois habitent la ville en 1965.

Longtemps soutenues et animées par les agriculteurs et les éléments de l'ancienne petite bourgeoisie, les caisses s'ouvrent graduellement à des populations devenues plus composites, suivant en cela le rythme imprimé par la croissance démographique des villes. L'examen du profil socioprofessionnel des sociétaires et des élus des caisses nouvellement fondées durant cette période témoigne de la diversification des bases sociales du mouvement. Dans l'ensemble, la composition sociale des assemblées de fondation reflète la montée du salariat. Plus de deux fondateurs sur trois dans les caisses érigées en milieu urbain après la guerre sont des salariés. Cette diversification des intérêts en présence trouve des échos à la direction des caisses alors que les représentants des nouvelles professions salariées chez les «cols blancs» viennent y concurrencer les positions détenues par les petits propriétaires et les membres des professions libérales. Portion quasi négligeable des élus locaux avant la fin de la guerre, les cols blancs salariés (cadres et administrateurs, spécialistes et employés réunis) forment plus du tiers des élus des caisses urbaines fondées après la guerre. Les caisses rurales établies durant la même période traduisent sensiblement le même phénomène alors que les cultivateurs voient leur poids relatif fléchir au profit d'autres groupes. Bien qu'ils y représentent encore la moitié des participants aux assemblées de fondation, les exploitants agricoles perdent la majorité qu'ils détenaient à la direction des trois conseils.

En somme, l'après-guerre est l'occasion pour les caisses d'enrichir la composition sociale de leur sociétariat et d'accueillir de nouveaux groupes à leur direction. Avant la guerre, la croissance du nombre de sociétaires était fonction de l'accroissement du nombre d'établissements. L'après-guerre est marqué davantage par l'enracinement et la consolidation des caisses existantes. En outre, les agents associés à la petite production, au petit commerce et aux professions

40 Les données relatives aux taux d'adhésion ne doivent pas être interprétées de façon absolue. Elles ont été calculées à titre indicatif. Elles ne tiennent pas compte du phénomène du cumul de comptes dans plus d'un établissement qui, croyons-nous, prend une importance croissante pendant cette période. Utilisées à titre comparatif, elles demeurent néanmoins une indication fiable de l'enracinement des caisses. 
libérales, qui voient leur poids diminuer sensiblement à la direction des caisses locales, doivent transiger avec de nouveaux groupes issus du monde ouvrier et des couches salariées de la petite bourgeoisie.

\section{UNE CROISSANCE SUSCITÉE PAR LES CAISSES URBAINES}

La croissance du mouvement des caisses pendant l'après-guerre procède aussi bien de la conjoncture économique que des nouveaux dynamismes qui se font jour en son sein. Dans le cadre d'une économie régionale entraînée par la croissance urbaine, elle s'appuie sur le développement des «grosses» caisses, favorise la consolidation de leur dispositif de gérance et contribue à l'érosion des bases paroissiales.

\section{Un développement inégal}

Si les chiffres pris dans leur ensemble laissent à l'observateur l'impression d'une croissance généralisée, continue et uniforme, un examen plus approfondi des données révèle l'existence d'importants écarts entre les caisses. Au sortir de la guerre, 17 des 129 caisses en activité (13\% des établissements) disposent de la moitié de l'actif cumulé à l'échelle régionale; les 89 caisses les plus petites (69\% des établissements) se partagent le quart de cet actif ${ }^{41}$. On pourrait attribuer ces disparités à la jeunesse d'un grand nombre de caisses à la fin de la guerre. Or cette tendance à la polarisation des ressources, loin de s'estomper, prend de l'ampleur. De plus, les inégalités de croissance n'ont que peu à voir avec le plus ou moins grand degré d'ancienneté des caisses. Ainsi, la moitié de l'actif réuni des 139 caisses en activité à l'échelle régionale est gérée par 16 d'entre elles (11,5\% des établissements) en 1965; le quart de cet actif est distribué parmi les 99 caisses les plus modestes (71\% des établissements). Les trois quarts de ces 16 «grosses» caisses ont été fondées entre 1936 et 1945. En comparaison, neuf des 17 caisses qui se partageaient la moitié de l'actif régional en 1945 avaient été fondées avant 1935. Il faut donc chercher ailleurs les facteurs d'explication de cette polarisation des ressources.

Une analyse plus serrée de l'évolution des caractéristiques du bloc des plus grosses caisses laisse entrevoir certains aspects significatifs de la réalité qui tend à s'imposer. D'abord, la croissance qui propulse le mouvement des caisses à compter de la Seconde Guerre nourrit des

41 Les chiffres présentés dans cette section ont été obtenus à partir d'un traitement statistique des données tirées des Rapports mensuels des caisses au 31 décembre, années 1945, $1950,1955,1960$ et 1965. 
disparités grandissantes entre les caisses urbaines et les caisses rurales; elle traduit la montée des premières et la perte de poids relatif des secondes. Treize des 30 caisses les plus importantes sur le plan financier sont implantées en milieu rural en 1945. En 1965, seulement deux caisses rurales figurent au nombre des 30 plus gros établissements. Les deux tiers de ces grosses caisses sont localisées sur la rive nord du Saint-Laurent, et cette répartition reste stable durant l'ensemble de la période. En fait, les nouveaux écarts entre les caisses ne se creusent plus tant en regard de leur territoire d'implantation ou de l'antériorité de la naissance des unes par rapport aux autres, comme c'était le cas durant la phase initiale, qu'à partir de leur appartenance à l'un ou l'autre des quatre pôles majeurs d'urbanisation dans la région. En 1945, la moitié des 30 caisses les plus importantes sur le plan financier sont localisées dans l'une ou l'autre des quatre grandes agglomérations suivantes: Trois-Rivières-Cap-de-la-Madeleine (6), ShawiniganGrand-Mère (3), Drummondville (4), Victoriaville (2). Vingt ans plus tard, ces quatre agglomérations sont le siège des activités de 25 des 30 plus grosses caisses affiliées à l'URTR. Il s'ensuit que la croissance du mouvement des caisses à compter de la Seconde Guerre est largement tributaire d'un développement régional favorisé par l'affirmation de quelques pôles urbains. L'envers de cette croissance, c'est la coexistence parfois difficile entre des caisses d'importance inégale alors que l'influence et le pouvoir dans l'organisation d'ensemble du mouvement se mesurent davantage à la valeur de l'actif qu'en fonction du nombre.

\section{L'autonomisation de la gérance locale}

Suivant le rythme de leur croissance, les caisses sont amenées à embaucher leur gérant sur une base permanente et à procéder à l'engagement d'employés. L'examen de la structure de la gérance locale et de la croissance du personnel des établissements pendant cette période permet de jeter un éclairage sur les situations fort variables d'une caisse à l'autre.

Jusqu'à la fin de la Deuxième Guerre mondiale, la fonction de gérant est assumée bénévolement ou à temps partiel dans la plupart des caisses. L'administration de la caisse, à partir du domicile du gérant, est souvent une affaire de famille. Les situations fréquemment rencontrées sont alors celles de gérants qui, appuyés de leur épouse ou d'autres proches, partagent leur temps entre la tenue de la caisse, leur commerce ou leurs autres activités professionnelles. Au milieu des années 1940,10\% des gérants de caisses sont bénévoles et seulement $6 \%$ le sont à temps plein; la grande majorité exercent la fonction en 
concurrence avec leurs activités professionnelles ${ }^{42}$. On ne relève alors qu'une moyenne régionale de deux employés par établissement en incluant les gérants: ces moyennes sont respectivement de 2,8 et 1,7 dans les caisses urbaines et les caisses rurales. C'est dire le caractère rudimentaire de l'organisation des caisses à la fin de la guerre.

La progression du volume des activités des caisses conjuguée à l'augmentation de leur chiffre d'affaires pendant l'après-guerre vont permettre à un nombre croissant de gérants de se consacrer entièrement à l'administration de leur établissement. Dans la région, quatre gérants sur dix dirigent leur caisse sur une base permanente au milieu des années 1960. C'est cependant dans les caisses urbaines que la gérance à temps plein tend à se généraliser. En 1965, les trois quarts de leurs gérants œuvrent à temps complet; cette proportion n'est que de $25 \%$ dans les caisses rurales. Généralement rémunérés en fonction de la valeur de l'actif, ils voient leur statut et leur avancement liés à la progression financière de leur établissement. La progression du personnel épouse sensiblement les mêmes tendances. Au milieu des années 1960, on recense en moyenne quatre employés par caisse en incluant les titulaires des postes de gérant. Encore une fois les situations se montrent sous des jours passablement différents selon les milieux ruraux et urbains. Dans le premier cas, la progression est minime: la moyenne d'employés par établissement y passe en effet de 1,7 à 2,8 entre 1945 et 1965 . Dans le second cas, l'accroissement est plus soutenu: la moyenne y est portée de 2,8 à 7,8 .

En somme, au cœur des années 1960, le mouvement des caisses s'apparente à une véritable mosaïque d'établissements. Les formes d'organisation peuvent être ramenées à deux archétypes. Le premier est encore le mode d'organisation prédominant dans la vaste majorité des caisses: il s'agit d'établissements fonctionnant selon des patterns de relations de travail similaires, à plusieurs égards, à ceux de la petite entreprise familiale. Le gérant, fréquemment à la tête d'un commerce ou au poste de secrétaire de la municipalité, de la commission scolaire ou d'une coopérative locale, dirige la caisse concurremment avec ses activités professionnelles. Le personnel s'y résume à un ou deux employés au plus, souvent l'épouse, le fils ou la fille du gérant. Dans

42 Parmi ces bénévoles, nous incluons les gérants qui ne bénéficient que d'une rémunération symbolique telle qu'un boni au terme de l'année financière ou toute autre rétribution dont la valeur ne vise de facto qu'à les dédommager (frais de déplacements, loyers, etc.). Il est à noter que les revenus que certains gérants à temps partiel tirent de la caisse sont parfois substantiels. Les données relatives à la gérance et au personnel des caisses présentées ici sont tirées d'un dépouillement préliminaire des rapports d'inspection d'un échantillon de 50 caisses représentatif de l'ensemble des caisses affiliées à l'Union régionale de Trois-Rivières. Cet aspect sera traité de façon plus approfondie dans une publication ultérieure. 
ces caisses, il n'est pas rare que le fils du gérant succède au père. Au moment où la majorité des établissements vivent encore à l'ère de la gestion familiale, un second groupe, appartenant principalement au monde urbain, est engagé dans une autre dynamique, plus sensible à la réalité marchande de l'environnement. Affranchis de leurs autres activités professionnelles, les gérants s'affairent à l'expansion de leurs caisses et aspirent à diriger des établissements financiers majeurs dans leurs milieux respectifs. Aussi, la gestion de ces «grosses» caisses commence-t-elle à se complexifier: les gérants s'adjoignent des assistants ou des comptables et l'amorce d'un mouvement en faveur de la syndicalisation des employés fait son apparition. La frontière entre l'ancienne et la nouvelle forme d'organisation n'est cependant pas aussi tranchée qu'il paraît à première vue. Ces formes coexistent de façon complexe et contradictoire, dans la majorité des établissements, selon des aménagements variables.

Le renforcement du dispositif de gérance locale, quoiqu'inégalement soutenu, apparaît donc comme l'un des traits marquants de l'évolution des caisses Desjardins pendant l'après-guerre. Il favorise l'émergence puis l'affirmation d'une nouvelle stratégie de développement plus agressive sur les marchés, dont les gérants des caisses les plus prospères sont les principaux promoteurs pendant les années 1950. Forts du poids économique représenté par leurs établissements, ces gérants exercent un pouvoir grandissant dans l'organisation d'ensemble du mouvement.

\section{L'érosion des bases paroissiales}

La dynamique de croissance qui tend à s'imposer à l'organisation des caisses dans l'après-guerre remet en cause l'un des fondements du modèle coopératif mis au point par A. Desjardins et soulignés à grands renforts par ses successeurs, à savoir sa filiation avec la paroisse. Cette concordance entre le territoire paroissial et l'aire d'activité de la caisse, dans l'esprit du fondateur, renvoyait au principe suivant lequel l'épargne, le crédit et les placements devaient servir le développement local. Elle reposait, d'autre part, sur une rationalité économique particulièrement originale qui plaçait la mutualité paroissiale au cœur de la régulation des rapports entre l'épargne et les risques associés au crédit. Par exemple, dans le cadre de politiques axées sur le primat de l'épargne, les mécanismes d'attribution du crédit étaient étroitement liés aux réseaux de sociabilité de la paroisse: exigence de la caution, critères relatifs à la moralité des emprunteurs, motifs d'emprunts, etc. Pendant les années 1950, deux mouvements, de provenance et de finalité contradictoire, sont à l'œuvre et contribuent de différentes façons à miner le caractère paroissial de la coopération d'épargne et de crédit. 
Le premier de ces mouvements, que nous ne traiterons pas ici, est venu des initiatives des Unions régionales et de la Fédération provinciale. En dépit de leur discours réaffirmant le caractère paroissial des caisses populaires, leurs activités sont allées dans le sens d'un renforcement de leurs capacités d'intervention dans les politiques des caisses, contribuant ainsi à «délocaliser» des pouvoirs et des activités jadis du ressort des caisses locales. Par ailleurs, elles ont mobilisé une partie croissante des fonds locaux dans le cadre du financement de leurs propres structures et des projets à caractère «national».

Le second mouvement est venu de la base de l'organisation, plus précisément des pressions des caisses urbaines les plus importantes sur le plan financier. La référence première de ces caisses n'est pas tant le développement de la paroisse que la concurrence que leur font les autres institutions financières. Au tournant de l'année 1950 est formé le Comité des grosses caisses, appelées «caisses millionnaires» ${ }^{43}$. Son existence se prolonge durant l'ensemble de la décennie alors qu'il voit à l'organisation de congrès annuels réservés aux seules «caisses millionnaires», indépendamment des instances fédérales du mouvement. Ces congrès sont l'occasion pour les caisses participantes de faire valoir les problèmes spécifiques auxquels elles sont confrontées, notamment en matière de crédit et de juridiction territoriale ${ }^{44}$. Les caisses millionnaires affiliées à l'URTR ont participé activement à ces congrès; certains de leurs gérants ont même été étroitement associés au Comité organisateur. La question de la limitation des activités des caisses au cadre paroissial est probablement celle qui a soulevé les tensions les plus vives entre les caisses «millionnaires» et les instances fédérales.

C'est dans les villes que la situation allait devenir la plus problématique. La poussée démographique du front urbain avait amené l'Église à révaluer sa stratégie d'implantation en y multipliant les paroisses, fractionnant au besoin le territoire des plus anciennes. Les caisses de première souche étaient évidemment réfractaires à l'idée de voir leur territoire d'activité amputé par l'implantation de nouvelles caisses dans les paroisses limitrophes. En pratique, elles ont déployé trois stratégies pour maintenir ou élargir leur base d'opération. Elles

43 L'appellation «caisse millionnaire» est empruntée au langage courant des dirigeants des caisses durant les années 1950. Elle servait alors à identifier les caisses les plus importantes en termes financiers, à savoir celles dont l'actif était supérieur à un million de dollars. Étant formé des gérants, le Comité des grosses caisses était aussi appelé Comité des gérants.

44 Nous avons abordé cette dimension ailleurs, Michel Bellefleur, Roger Levasseur et Yvan Rousseau. op. cit. 
ont d'abord résisté à l'établissement de nouvelles caisses sur leur territoire initial. Elles ont aussi sollicité les sociétaires ou les résidants des paroisses voisines, exercant ainsi une concurrence jugée déloyale aux petites caisses par des taux d'intérêt plus avantageux sur l'épargne et le crédit. Enfin, durant la première moitié des années 1950, elles contestent ouvertement la limitation de leurs activités au cadre paroissial, faisant parvenir, devant le refus des instances fédérales, des demandes d'extension de territoire au sous-secrétaire de la province ${ }^{45}$. Les caisses millionnaires perçoivent la restriction de leurs activités au territoire paroissial comme une atteinte à leur autonomie et comme une centralisation excessive du pouvoir entre les mains des fédérations.

Les débats relatifs au cadre paroissial vont se prolonger au-delà des années 1960. Mais avec les années, une plus grande perméabilité des limites territoriales des caisses se confirmera, surtout dans les milieux urbains. Les instances fédérales assoupliront progressivement leur position à l'égard des caisses urbaines, évoquant la mobilité des populations de ces milieux ${ }^{46}$. Le congrès de 1965 est par ailleurs l'occasion pour les participants de s'interroger sur la pertinence de la paroisse comme cadre territorial de la caisse populaire. Dans son allocution d'ouverture, le président de la Fédération provinciale, Émile Girardin, ouvrait les discussions sur la possibilité de regrouper des sociétaires sur une base professionnelle plutôt que paroissiale, et soulevait l'éventualité de fusionner certaines caisses afin de consolider l'actif des plus petites ${ }^{47}$. Le second choix sera retenu. Les fusions de caisses et l'ouverture de comptoirs pendant les années 1970-1980 viennent confirmer les orientations prises au milieu des années 1960.

\section{CONCLUSION}

La première phase d'existence du mouvement des caisses coïncide avec l'arrivée du $\mathrm{XX}^{\mathrm{e}}$ siècle et se prolonge jusqu'au milieu des années trente. Cette période est globalement marquée par l'influence d'Alphonse Desjardins, de ses proches collaborateurs et de ses successeurs immédiats parmi lesquels figurent notamment les repré-

45 Lettre du sous-secrétaire de la province à la Fédération de Québec des Unions régionales, Québec, 11 mai 1955. Voir également la conférence de Cyrille Vaillancourt intitulée «Comment une caisse peut-elle, en pratique, dévier de l'esprit du Commandeur Desjardins? Quels seraient les moyens efficaces d'éducation populaire qui conserveraient à nos caisses l'esprit du fondateur?», Compte rendu du cinquième congrès des caisses populaires importantes, tenu à Sherbrooke les 7, 8 et 9 mai 1956, 10-29.

46 FQUR, Procès-verbal du Conseil d'administration, 3 octobre 1965.

47 La caisse populaire et la communauté québécoise en 1965, Rapport des travaux du 9. congrès des caisses populaires, tenu à Montréal du 14 au 18 juin 1965, $147 \mathrm{p}$. 
sentants du clergé. Les caisses s'apparentent alors à un mouvement à dominante rurale inégalement développé, encore peu intégré malgré leur regroupement à l'échelle régionale puis nationale durant l'entredeux-guerres, et particulièrement sensible aux fluctuations de l'économie régionale. Cette phase pionnière de l'existence des caisses est également celle d'un mouvement recrutant ses effectifs parmi les agents de la petite propriété (agriculteurs, artisans, marchands) et les membres des professions libérales et du clergé. En dépit des efforts déployés par les leaders régionaux pour propager la formule des caisses populaires, elles demeurent encore peu enracinées dans les collectivités locales. En 1940, elles ne parviennent toujours pas à rassembler plus de $10 \%$ des résidants des localités desservies et le crédit n'y est accessible qu'à une infime minorité. Le projet porté par les leaders de première souche va reposer sur trois orientations constamment réaffirmées durant l'entre-deux-guerres: l'organisation de base fondée sur la paroisse, l'initiative laissée aux élites locales pour assurer la diffusion et le fonctionnement des caisses ainsi que le rôle central qu'elles sont appelées à jouer dans le monde agricole. Ces trois axes de la mutualité d'épargne et de crédit telle qu'elle était véhiculée par les dirigeants de la première heure ont convergé vers la promotion d'une forme d'organisation de la vie sociale apparentée à la sociabilité villageoise.

Dans une société qui s'urbanise et s'industrialise, la concentration du pouvoir économique remet en cause ce projet qui se propose de revitaliser la petite propriété et la société rurale. En l'espace d'une dizaine d'années, soit du milieu des années 1930 à la fin de la Seconde Guerre, la configuration des caisses est profondément bouleversée. Le temps II de l'évolution du sociétariat se déroule sous le signe de la transition alors que le mouvement gagne en étendue en quadrillant aussi bien l'espace urbain que rural, profitant ainsi de la conjoncture favorable de la Seconde Guerre. Cette époque charnière de l'évolution du mouvement est notamment marquée par son ouverture à la ville et son rythme de croissance inégalé tant sur le plan du sociétariat que sur celui des assises financières. Bien que plus diversifiées dans leurs bases sociales et leur direction, les caisses n'en continuent pas moins à s'appuyer sur le leadership du monde agricole et de la petite bourgeoisie locale. L'autonomie locale, le renforcement des pouvoirs fédéraux et les ambitions de certaines caisses en pleine expansion sont au nombre des enjeux qui pointent au sortir de la guerre.

La période qui s'amorce avec la fin du conflit mondial et se prolonge jusqu'aux années 1960 est celle d'un mouvement qui gagne en profondeur, qui s'enracine dans les paroisses. Peu de nouvelles caisses voient le jour comparativement à la période précédente, 
cependant que les caisses existantes vont accroître leur sociétariat de façon marquée. Si les données prises globalement laissent l'impression d'une croissance linéaire, continue et généralisée, une analyse plus serrée révèle des disparités considérables. En effet, les années qui vont de l'après-guerre à la Révolution tranquille sont marquées par un développement inégal ainsi que par une diminution constante du poids relatif des caisses rurales dans l'organisation d'ensemble du mouvement. Ce temps III de l'évolution des caisses est celui d'un mouvement appuyé sur la croissance urbaine. La concurrence plus vive avec les autres institutions financières mais aussi entre caisses et les pressions des «grosses» caisses sur le cadre paroissial témoignent à différents degrés d'une évolution qui participe de plus en plus d'une nouvelle dynamique de développement. Pendant ce temps, les agents de la petite propriété et les représentants des professions libérales conservent leur importance à la direction des caisses locales, mais ils doivent désormais composer avec de nouveaux groupes issus de l'élargissement des bases sociales des caisses, du renforcement de la gérance locale et de la consolidation des pouvoirs fédéraux. 